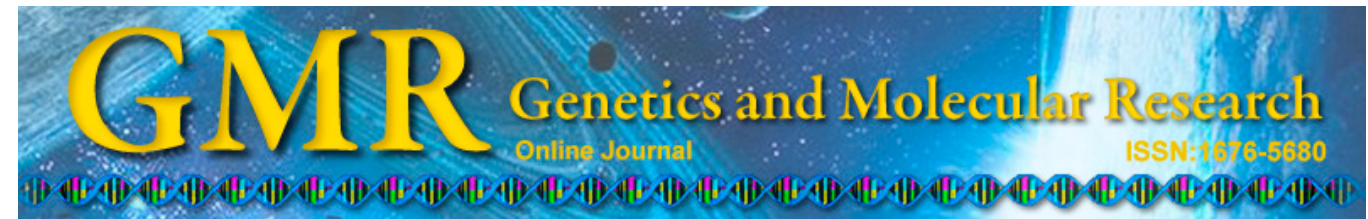

\title{
Weak cation magnetic separation technology and MALDI-TOF-MS in screening serum protein markers in primary type I osteoporosis
}

\author{
X.L. Shi ${ }^{1 *}$, C.W. Li ${ }^{2 *}$, B.C. Liang ${ }^{3 *}$, K.H. He ${ }^{3}$ and X.Y. $\mathrm{Li}^{3}$ \\ ${ }^{1}$ Department of Osteology, \\ The Second Affiliated Hospital of Zhejiang Chinese Medical University, \\ Hangzhou, China \\ ${ }^{2}$ Department of Diagnostics of Traditional Chinese Medicine, \\ College of Basic Medical Science, Zhejiang Chinese Medical University, \\ Hangzhou, China \\ ${ }^{3}$ The Second Clinical Medical College, Zhejiang Chinese Medical University, \\ Hangzhou, China \\ *These authors contributed equally to this study. \\ Corresponding author: X.L. Shi \\ E-mail: xlshi-2002@163.com
}

Genet. Mol. Res. 14 (4): 15285-15294 (2015)

Received July 3, 2015

Accepted October 19, 2015

Published November 30, 2015

DOI http://dx.doi.org/10.4238/2015.November.30.4

\begin{abstract}
We investigated weak cation magnetic separation technology and matrix-assisted laser desorption ionization-time of flight-mass spectrometry (MALDI-TOF-MS) in screening serum protein markers of primary type I osteoporosis. We selected 16 postmenopausal women with osteoporosis and nine postmenopausal women as controls to find a new method for screening biomarkers and establishing a diagnostic model for primary type I osteoporosis. Serum samples were obtained from controls and patients. Serum protein was extracted with the WCX protein chip system; protein fingerprints were examined using MALDI-TOF-MS. The preprocessed and model construction data were handled by the ProteinChip system. The diagnostic models were
\end{abstract}


established using a genetic arithmetic model combined with a support vector machine (SVM). The SVM model with the highest Youden index was selected. Combinations with the highest accuracy in distinguishing different groups of data were selected as potential biomarkers. From the two groups of serum proteins, 123 cumulative MS protein peaks were selected. Significant intensity differences in the protein peaks of 16 postmenopausal women with osteoporosis were screened. The difference in Youden index between the four groups of protein peaks showed that the highest peaks had mass-to-charge ratios of 8909.047, $8690.658,13745.48$, and 15114.52. A diagnosis model was established with these four markers as the candidates, and the model specificity and sensitivity were found to be $100 \%$. Two groups of specimens in the SVM results on the scatterplot were distinguishable. We established a diagnosis model, and provided a new serological method for screening and diagnosis of osteoporosis with high sensitivity and specificity.

Key words: Primary type I osteoporosis; Proteomics;

Weak cation magnetic separation technology; Biomarker;

Matrix-assisted laser desorption ionization-time of flight-mass spectrometry

\section{INTRODUCTION}

Osteoporosis is a systemic metabolic bone disease characterized by low bone mass and bone microstructure degeneration. With the advent of the aging society, there has been a rapid upward trend in the incidence of osteoporosis, which is a serious threat to the health of the elderly (Czerwiński et al., 2007). According to one survey, the incidence of osteoporosis was $31.2 \%$ in men ( 15 million) and $10.4 \%$ in women (54.1 million) totaling about 69.1 million out of a population of Chinese patients over the age of 50. Currently, China is considered to be one of the largest regions in terms of the number of osteoporosis patients in the world. However, the incidence rate was significantly higher for postmenopausal women than for men. In most women, the rate of bone loss increases for several years after menopause, then slows down again, but continues. The results of one study suggest that estrogen deficiency, aging, and calcium deficiency are the main causes of the increased rate of bone loss and bone turnover in postmenopausal women. Therefore, primary type I osteoporosis, which has become a serious public health concern, receives a great deal of attention (Wark, 1996). Osteoporosis is a skeletal disorder characterized by compromised bone strength, which predisposes the individual to an increased risk of fractures of the hip, spine, and other skeletal sites. Osteoporosis fractures have the most serious consequences.

Dual-energy X-ray absorptiometry, which measures bone mineral density (BMD), is currently recognized as the gold standard of osteoporosis diagnostic (Kanis, 2002). However, BMD only reflects bone mineral content, and not all the information about the bone such as bone mass and bone microstructures. Early symptoms of osteoporosis are not easy to detect, and therefore many patients are often only diagnosed after the first fracture. BMD cannot detect early osteoporosis. Moreover, BMD does not reflect change of bone matrix, bone turnover, or bone strength, and cannot accurately predict the risk of bone fracture (Sone, 2011).

With the completion of the Human Genome Project, and the arrival of the post-ge- 
nomic era, research on the structure and function of proteins can directly elucidate physiological and pathological mechanisms. We can rapidly screen the specific biomarkers of diseases using proteomics technologies (Cho, 2007), clarify the pathogenesis of the disease, and explore new methods of treatment.

Matrix-assisted laser desorption ionization-time of flight-mass spectrometry (MALDITOF-MS) is a recently emerged proteomics method, with the potential to detect various clinical samples such as serum, urine, pleural effusion, ascites, and a number of secretions (Li et al., 2009).

In this study, we introduced a newly developed mass spectrometry-based technology: weak cation magnetic separation technology and MALDI-TOF-MS (Dai et al., 2010). We searched for efficient serum protein biomarkers, and attempted to predict the molecular mechanisms of primary type I osteoporosis, thereby reducing the uncertainties and potential risks for primary type I osteoporosis patients.

\section{MATERIAL AND METHODS}

\section{Subjects and samples collection}

Sixteen patients with primary type I osteoporosis at the Department of Orthopedics, The Second Affiliated Hospital of Zhejiang Chinese Medicine University were enrolled in the study between June 2012 and January 2013. Nine healthy postmenopausal women with normal bone mass ranges served as controls.

\section{Diagnostic criteria}

Osteoporosis was diagnosed according to the World Health Organization's recommended criterion: the lumbar vertebra normal position bone density was surveyed using dual energy X-ray absorptiometry (Vestergaard et al., 2005; Unnanuntana et al., 2010); compared with a normal adult of the same gender and race, $\mathrm{T} \leq-2.5$ could be diagnosed as osteoporosis, where $\mathrm{T}$ $=($ the standard deviation of measured value - peak bone mass $) /$ normal adult bone density.

\section{Inclusion criteria}

Patients were included in the study based on the following criteria: i) they conformed to the osteoporosis diagnostic criteria; ii) they were postmenopausal women; and iii) they were between 50 and 70 years old.

\section{Exclusion criteria}

Patients were excluded from the study based on the following criteria: i) those that also had diseases that severely affect the metabolism of bone or calcium, such as diabetes, Cushing's syndrome, function changing of the thyroid or parathyroid, osteomalacia, rheumatoid arthritis, multiple myeloma, bone tumor, osteoarthrosis, Paget's disease, and osteogenesis imperfecta; ii) those that also had severe primary cardiac diseases, or diseases of the cerebral vessels or hematopoietic system; iii) those that also had severe liver function or renal insufficiencies; iv) those taking drugs within the past 6 months that affect bone metabolism, such 
as estrogen, steroid hormones, calcitonin, parathyroid hormones, bisphosphonates, fluoride, vitamin $\mathrm{D}$, anticonvulsant drugs, and diuretics; v) those who had a medical history of mental illness; and vi) patients with Alzheimer's disease.

\section{Ethical review}

This study was approved by the local Ethics Committee of The Second Affiliated Hospital of Zhejiang Chinese Medicine University. The patients and volunteers provided written informed consent for their participation.

\section{Sample collection and preparation}

Blood samples $(5 \mathrm{~mL})$ were collected in the morning and allowed to clot at room temperature for $1-2 \mathrm{~h}$. The samples were then centrifuged at $4^{\circ} \mathrm{C}$ for $5 \mathrm{~min}$ at $943 \mathrm{~g}$. The serum was frozen and stored at $-80^{\circ} \mathrm{C}$ for future analysis.

\section{Weak cation magnetic separation technology analysis}

We prepared a $200-\mu \mathrm{L}$ sample tube of thoroughly mixed weak cation magnetic suspension by adding $10 \mu \mathrm{L}$ magnetic beads binding buffer, $10 \mu \mathrm{L}$ magnetic beads suspension, and $5 \mu \mathrm{L}$ serum, mixing at least 5 times using the sampling gun, and standing at room temperature for $5 \mathrm{~min}$. We put the sample tube into the magnetic separator, maintained the magnetic field for $1 \mathrm{~min}$, used the sampling gun to absorb the liquid after separation of the magnetic beads and liquid, and added to the sample tube $100 \mu \mathrm{L}$ magnetic beads cleaning buffer. We then moved the sample tube 10 times between the two adjacent holes of the magnetic separator, left to stand, and used the sample gun to absorb the supernatant again after magnetic beads adherence. We repeated the cleaning process twice. We then took down the sample tube from the magnetic separator, added $5 \mu \mathrm{L}$ magnetic beads elution buffer to the sample tube, and repeated the pipetting. We put the sample tube into the magnetic separator and let it stand for $2 \mathrm{~min}$, transferred the supernatant to a clean $0.5-\mathrm{mL}$ sample tube when the magnetic beads were completely adhered, then added $5 \mu \mathrm{L}$ magnetic beads stabilizing buffer, dotted a $1-\mu \mathrm{L}$ sample on the target after carefully pipetting and mixing using the sampling gun, and added $10 \mu \mathrm{L}$ matrix solution after drying. Finally, after drying again, we carried out the protein mass spectrometry detection.

\section{MALDI-TOF-MS}

MALDI-TOF-MS parameters are detailed in Table S1.

\section{Detection data analysis}

The Zhejiang University-ProteinChip data analysis system (ZJU-PDAS) software, designed at Zhejiang University Cancer Institute, was used to analyze the raw data (Qiu et al., 2009). The process was as follows. i) The original spectrum was uploaded to the server to map data for protein analysis, and to process homogeneous data. ii) We removed the mass-tocharge ratio $(\mathrm{m} / \mathrm{z})$ of raw data below peak 2000 , used the undecimated discrete wavelet trans- 
form analysis method to remove the noise caused by the mass spectrometer itself, and used the amended data after the removal of the baseline noise spectrum and correction of the molecular weight values map. iii) We identified the protein charge ratio peaks with local minima, and filtration peak signal-to-noise ratios of less than 3 . The differences in $\mathrm{m} / \mathrm{z}$ between each sample of $<0.3 \%$ of the peak were clustered together. The percentage of each peak appears in spectra is specified to 10 . The matched peak across spectra is defined as peaks cluster. iv) The normalization is performed only by the identified peak clusters. v) After pretreatment, we filtered out protein charge ratio peaks using the Wilcoxon rank sum test, and screened differential protein peaks $(\mathrm{P}<0.05)$. vi) We screened a random combination of different proteins, and used a genetic arithmetic model and the support vector machine (SVM) computing model to establish serum protein fingerprints.

For the SVM, we used the radial basis function, a gamma value of 0.6 , and the penalty function (C) was set to 19. The highest elected combinations were used to build the SVM model to predict the Youden index. Because the final candidate signs matter, we used the leave-one-assessment model to predict the effect of an established law and the final results were cross-validated. ZJU-PDAS-specific parameters of analysis are detailed in Table S2.

\section{Statistical analysis}

We used the SPSS13.0 software to process the baseline data from the two groups of subjects, comparing age, weight, height, and duration of menopause in the two groups using the Student $t$-test with a test level of $\alpha=0.05$. We used ZJU-PDAS for homogeneity analysis and data on protein patterns, and the Wilcoxon rank sum test (test level of $\alpha=0.05$ ) to screen out the different protein peak intensities of the two groups of specimens after the homogenization processing of the protein peak intensities of the two groups of specimens of serum protein. We combined the differential protein peaks randomly, and used a genetic algorithm (GA) (Deb et al., 2002) and SVM (Suykens and Vandewalle, 1999) for further analyses. We established the diagnosis model and the combination of the highest Youden index of the SVM model prediction as the candidate marker, and verified the discriminant effect model by leaving-one-out and cross-validation methods (Ney et al., 1995).

\section{RESULTS}

\section{Statistical analysis of baseline data}

There were no statistically significant differences in age, weight, height, and duration of menopause between the two groups of subjects $(\mathrm{P}>0.05)$. The results are shown in Table 1 .

Table 1. Comparison of baseline data from the two groups of postmenopausal women.
\begin{tabular}{lcccc}
\hline Group & Age (years) & Weight $(\mathrm{kg})$ & Height $(\mathrm{cm})$ & Duration of menopause (years) \\
\hline Osteoporosis & $61.32 \pm 6.41$ & $54.04 \pm 5.15$ & $151.46 \pm 10.07$ & $5.34 \pm 2.53$ \\
Controls & $58.00 \pm 3.48$ & $51.24 \pm 3.26$ & $158.52 \pm 11.61$ & $4.89 \pm 1.45$ \\
$t$ & 4.022 & 4.371 & 4.151 & 4.017 \\
P values & 0.062 & 0.053 & 0.059 & 0.069 \\
\hline
\end{tabular}

Data shown are reported as means \pm standard deviation. 


\section{Detection results}

One hundred and twenty-three cumulative mass spectrometry protein peaks were detected from the specimens of serum proteins from the two groups. The results are shown in Figure 1. Although 16 peaks showed differences between the two groups $(\mathrm{P}<0.05)$, only four $(8909.047,8690.658,13,745.48$, and $15,114.52 \mathrm{Da})$ showed statistically significant differences by further analysis using the highest Youden index (Fluss et al., 2005; Ruopp et al., 2008). The results are shown in Table 2, which presents the statistics. The diagnosis model with the four markers as the candidate proteins was established. Figure 2A-D show peaks with statistically significant differences at $8909.047 \mathrm{~m} / \mathrm{z}, 8690.658 \mathrm{~m} / \mathrm{z}, 13,745.48 \mathrm{~m} / \mathrm{z}$, and $15,114.52 \mathrm{~m} / \mathrm{z}$, respectively. Specificity and sensitivity of the model were $100 \%$. The results are shown in Table 3. Two groups of specimens could be clearly distinguished in the SVM discriminant results scatter diagram. The results are shown in Figure 3.

All four peaks showed much greater expression in the 16 patients than in the 9 patients; Wilcoxon tests using the Youden index for all four peaks produced $\mathrm{P}$ values $<0.05$. The four peaks established a protein pattern for screening osteoporosis serum protein markers in the primary type I osteoporosis patients.

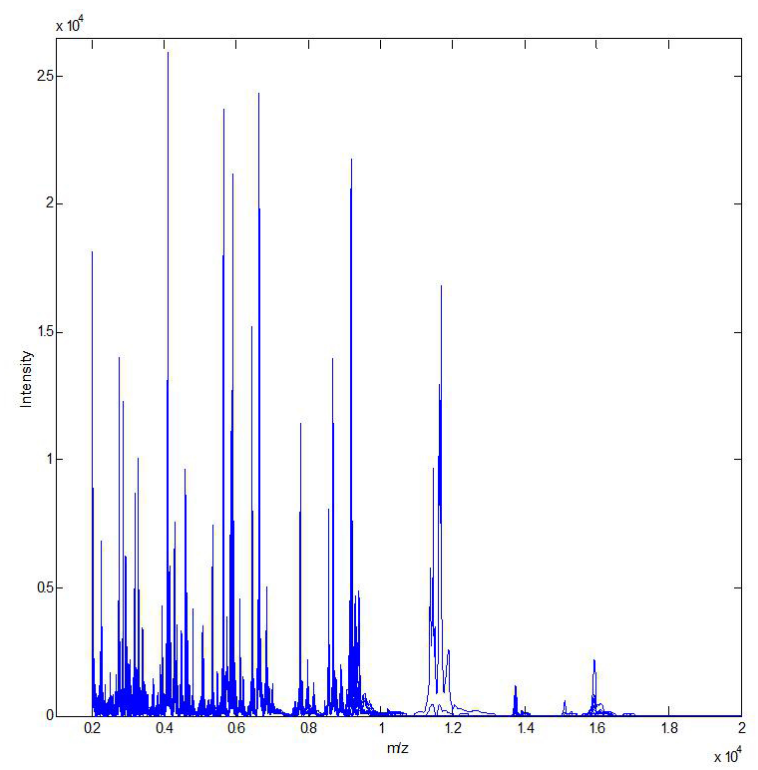

Figure 1. Distribution intention of cumulative mass spectrometry protein peaks of all samples.

Table 2. Statistics from the serum protein peaks detected in the two groups.

\begin{tabular}{lcrc}
\hline Mass-to-charge ratio & Osteoporosis & Controls & P value \\
\hline 8909.047 & $1315.214 \pm 395.182$ & $904.730 \pm 282.261$ & 0.012 \\
8690.658 & $8685.821 \pm 3252.091$ & $5888.973 \pm 1716.977$ & 0.026 \\
$15,114.520$ & $201.115 \pm 261.768$ & $78.747 \pm 142.429$ & 0.027 \\
$13,745.480$ & $518.442 \pm 308.736$ & $277.820 \pm 207.656$ & 0.049 \\
\hline
\end{tabular}

Data shown are reported as means \pm standard deviation.

Genetics and Molecular Research 14 (4): 15285-15294 (2015) ～CFUNPEC-RP www.funpecrp.com.br 

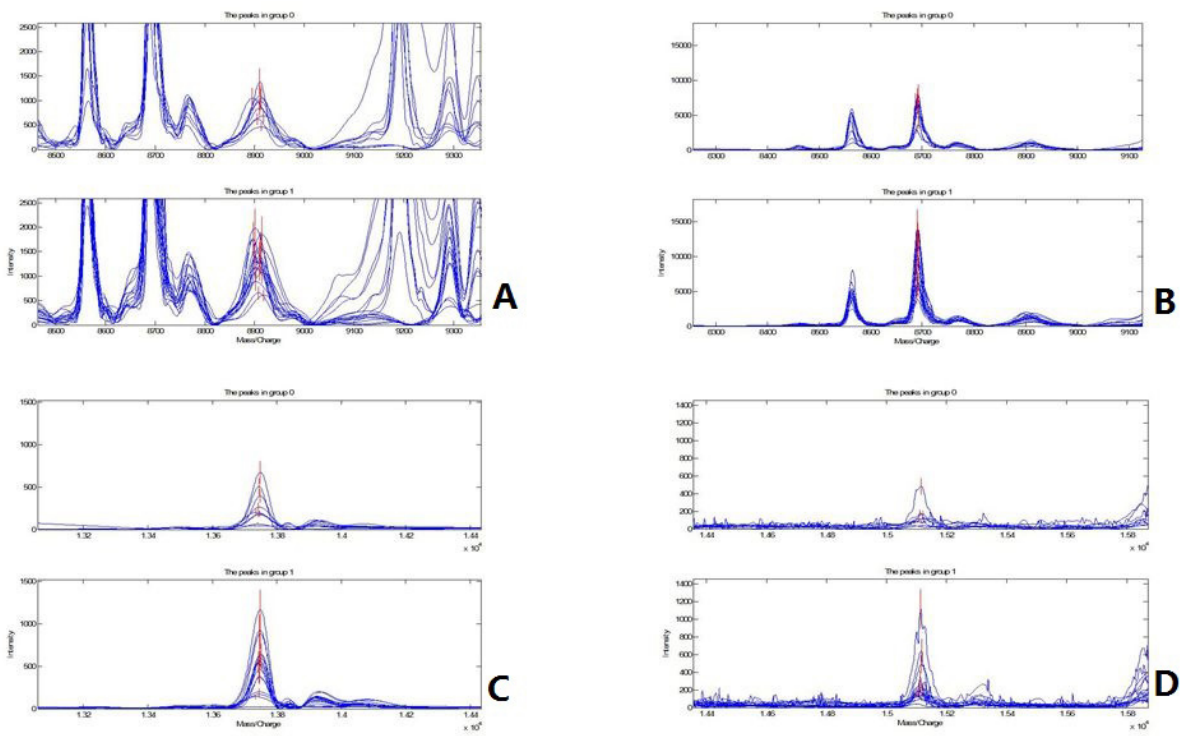

Figure 2. Comparison of the four peaks of statistically significant differences at $8909.047 \mathrm{~m} / \mathrm{z}$ (A); $8690.658 \mathrm{~m} / \mathrm{z}$ (B); $13,745.480 \mathrm{~m} / \mathrm{z}(\mathbf{C})$; and $15,114.52 \mathrm{~m} / \mathrm{z}$ (D) among the two groups. $(\mathrm{P}<0.05) . \mathrm{m} / \mathrm{z}=$ mass/charge; group 0 means the control group; group 1 means the osteoporosis group.

\section{Table 3. Results of diagnosis model cross-validation.}

\begin{tabular}{lccc}
\hline Group & Osteoporosis & Controls & Total \\
\hline Osteoporosis & 16 & 0 & 16 \\
Normal control & 0 & 9 & 9 \\
Total & 16 & 9 & 25 \\
\hline
\end{tabular}

The specificity of the model was $100 \%$; the sensitivity was $100 \%$.

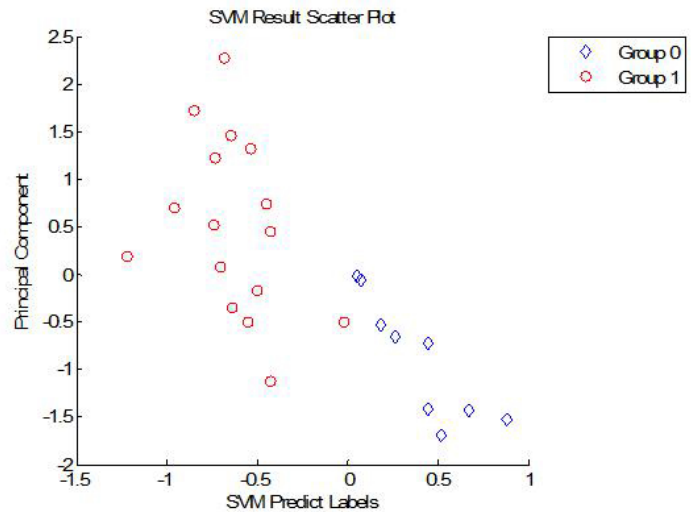

Figure 3. Serum specimen scatter diagram of support vector machine (SVM) discriminant results among the two groups. In the distribution map of the two groups, each point represents a sample; the vertical coordinate is the main component and the abscissa is the predicted value. The two groups can be clearly distinguished. $\mathrm{m} / \mathrm{z}=$ mass $/$ charge; group 0 means the control group; group 1 means the osteoporosis group. 


\section{Bioinformatic identification of differences in the protein peaks}

We used the TagIdent tool network software (http://web.expasy.org/tagident/) (formerly GuessProt) (Wilkins et al., 1998; Zheng et al., 2003). The molecular weight range was set to $0.3 \%$. The isoelectric point range value was set to $\min =4, \max =14$. We checked for protein sequences using oxidized cysteines (-SS-). The organism name was Homo sapiens. Only the sequences matching the tag were displayed. We displayed the predicted $\mathrm{N}$-terminal sequence using databases on UniProtKB/Swiss-Prot. Protein candidates were selected based on similarity of molecular weight.

\section{DISCUSSION}

The occurrence of osteoporosis in postmenopausal women is about 30\% (Ettinger et al., 1999; Siris et al., 2001). Serum marker screening of high specificity and sensitivity for the prevention and treatment of postmenopausal osteoporosis is important. BMD is the diagnostic gold standard for osteoporosis. Currently, clinical experts believe that hormone secretion is the main cause of osteoporosis after menopause. In recent years, with the development of proteomics technology, a variety of biological markers have been widely used in clinical settings. Biological markers play an important role in disease diagnosis. Using serum proteomics technology (Coombes et al., 2005) to find important molecular markers is an effective method for the clinical diagnosis of the disease.

Currently, the commonly used serum bone markers such as total procollagen type I amino-terminal propeptide (Chen et al., 2005), the carboxy-terminal cross-linking telopeptide of type I collagen ( $\beta$-CrossLaps), N-terminal osteocalcin, and 25-hydroxy vitamin D3 are used to detect changes in bone metabolism. However, some indicators of bone markers have low sensitivity and specificity in the early diagnosis of osteoporosis, and do not meet the requirements of non-invasiveness in clinical practice. At the same time, some other indicators cannot distinguish between markers in postmenopausal osteoporosis health (Bhattacharyya et al., 2008).

In this study, all aspects of data processing, such as sample inclusion and exclusion, serum collection, processing and storage of serum, serum protein separation and purification, sample point targeting, mass spectrometry acquisition, experimental methods, and parameter settings, were optimized. Discovery researches in the clinical samples is better than in vitro and in vivo experiments.

Magnetic beads, also known as nano-magnetic beads, usually consist of a magnetic core and a polymer shell outside the core. The magnetic beads can be positioned in a magnetic field, separated from the surrounding medium. By modifying the surface of the microsphere beads, magnetic beads can be coupled with a variety of biologically active substances such as antibodies, antigens, receptors, enzymes, and nucleic acids. The bioactive substance can be immobilized on the magnetic beads (Fiedler et al., 2007).

MALDI-TOF-MS is a widely used proteomics technology with several advantageous features. The MALDI-TOF-MS method can identify cells, tissues, or whole body proteins, and provides a set of information on protein function and mode.

Bruker's Microflex type MALDI-TOF-MS allows the use of crude samples of minimal size, and is large-scale, ultra-volume, high-throughput, and automated. Not only can it find a protein or biological molecular marker, it also detects combinations of proteins existing 
in different forms (Gould et al., 2004). Weak cation magnetic separation technology combined with MALDI-TOF-MS does not require adjustments of the mixed solution $\mathrm{pH}$, temperature, ionic strength, or dielectric constant, and can avoid the loss of protein that is a disadvantage of the traditional separation process, with fast, high-purity, and high-yield results.

In this study, a data analysis software system was used. The noise was removed using undecimated discrete wavelet transform analysis (Aiazzi et al., 2002). A serum protein fingerprinting model was established by GA combined with SVM operations. GA showed good parallelism and global optimization by simulating the evolution of life; SVM exhibited many unique advantages in solving small sample, nonlinear, and high-dimensional pattern recognition problems, and achieved the statistical theory of structural risk minimization principle. The application of these techniques in bioinformatics data processing and modeling produced a serum protein fingerprint diagnostic model with high credibility.

The application of weak cation magnetic separation technology combined with the MALDI-TOF-MS and the ZJU-PDAS software screened the differential protein peaks of the primary type I osteoporosis and healthy postmenopausal women.

GA combined with SVM was used to establish the discriminant model. Leave-one-out cross-validation verified the determination results of the discriminant model. Bioinformatic analysis and statistical processing were used to screen a combination of four protein peaks, which were established by analysis of the highest Youden index theory. Four proteins biomarker (m/z: 8909.047, 8690.658, 13,745.480, and 15,114.520 Da) were screened. The validated diagnostic sensitivity of the model was $100 \%$, and the specificity was also $100 \%$. The accuracy and rigor of the diagnostic model were better than the previous modeling program. Early diagnosis using accurate screening and sensitive biomarkers is very important in the treatment of primary type I osteoporosis. Molecular weights were fed into the TagIdent tool network software and four candidate proteins were obtained (8909.047 Da matched the C-C motif of chemokine 23; 8690.658 Da matched the C-C motif of chemokine 2; 13,745.480 Da matched leukocyte cell-derived chemotaxin 1; and 15,114.52 Da matched gamma-parvin).

This study provides a new serological method for the screening and diagnosis of primary type I osteoporosis using serum protein markers. It will help clinicians in the correct diagnosis and treatment of the disease. However, there is still a gap between our findings and the application of the technique in clinical practice. Future multi-center, controlled studies with larger sample sizes are required to determine the sensitivity and specificity of the five identified serum biomarkers in the diagnosis of osteoporosis. Verification of the functions, interactions, and metabolic pathways of these proteins should be the focus of future research.

\section{Conflicts of interest}

The authors declare no conflict of interest.

\section{ACKNOWLEDGMENTS}

Research supported by the Natural Science Fund Project of Zhejiang Province in 2012 (\#LY12H290037); the National Natural Science Foundation of China in 2013 (\#2013 81373878); and the Natural Science Foundation of Zhejiang Province in 2013 (\#LY13H290009). 


\section{Supplementary material}

\section{REFERENCES}

Aiazzi B, Alparone L, Baronti S and Garzelli A (2002). Context-driven fusion of high spatial and spectral resolution images based on oversampled multiresolution analysis. IEEE Geosci. Remote S. 40: 2300-2312.

Bhattacharyya S, Siegel ER, Achenbach SJ, Khosla S, et al. (2008). Serum biomarker profile associated with high bone turnover and BMD in postmenopausal women. J. Bone Miner. Res. 23: 1106-1117.

Chen P, Satterwhite JH, Licata AA, Lewiecki EM, et al. (2005). Early changes in biochemical markers of bone formation predict BMD response to teriparatide in postmenopausal women with osteoporosis. J. Bone Miner. Res. 20: 962-970.

Cho WC (2007). Proteomics technologies and challenges. Genomics Proteomics Bioinformatics 5: 77-85.

Coombes KR, Morris JS, Hu J, Edmonson SR, et al. (2005). Serum proteomics profiling - a young technology begins to mature. Nat. Biotechnol. 23: 291-292.

Czerwiński E, Badurski JE, Marcinowska-Suchowierska E and Osieleniec J (2007). Current understanding of osteoporosis according to the position of the World Health Organization (WHO) and International Osteoporosis Foundation. Ortop. Traumatol. Rehabil. 9: 337-356.

Dai Y, Hu C, Wang L, Huang Y, et al. (2010). Serum peptidome patterns of human systemic lupus erythematosus based on magnetic bead separation and MALDI-TOF mass spectrometry analysis. Scand. J. Rheumatol. 39: 240-246.

Deb K, Pratap A, Agarwal S and Meyarivan T (2002). A fast and elitist multiobjective genetic algorithm: NSGA-II. Evol. Comp. IEEE Trans. 6: 182-197.

Ettinger B, Black DM, Mitlak BH, Knickerbocker RK, et al. (1999). Reduction of vertebral fracture risk in postmenopausal women with osteoporosis treated with raloxifene: results from a 3-year randomized clinical trial. Multiple Outcomes of Raloxifene Evaluation (MORE) Investigators. JAMA 282: 637-645.

Fiedler GM, Baumann S, Leichtle A, Oltmann A, et al. (2007). Standardized peptidome profiling of human urine by magnetic bead separation and matrix-assisted laser desorption/ionization time-of-flight mass spectrometry. Clin. Chem. 53: 421-428.

Fluss R, Faraggi D and Reiser B (2005). Estimation of the Youden Index and its associated cutoff point. Biom. J. 47: 458-472.

Gould KL, Ren L, Feoktistova AS, Jennings JL, et al. (2004). Tandem affinity purification and identification of protein complex components. Methods 33: 239-244.

Kanis JA (2002). Diagnosis of osteoporosis and assessment of fracture risk. Lancet 359: 1929-1936.

Li YZ, Hu CJ, Leng XM, Zhao GF, et al. (2009). Promising diagnostic biomarkers for primary biliary cirrhosis identified with magnetic beads and MALDI-TOF-MS. Anat. Rec. 292: 455-460.

Ney H, Essen U and Kneser R (1995). On the estimation of 'small' probabilities by leaving-one-out. IEEE Pattern Anal. 17: 1202-1212.

Qiu FM, Yu JK, Chen YD, Jin QF, et al. (2009). Mining novel biomarkers for prognosis of gastric cancer with serum proteomics. J. Exp. Clin. Cancer Res. 28: 126

Ruopp MD, Perkins NJ, Whitcomb BW and Schisterman EF (2008). Youden Index and optimal cut-point estimated from observations affected by a lower limit of detection. Biom. J. 50: 419-430.

Siris ES, Miller PD, Barrett-Connor E, Faulkner KG, et al. (2001). Identification and fracture outcomes of undiagnosed low bone mineral density in postmenopausal women: results from the National Osteoporosis Risk Assessment. JAMA 286: $2815-2822$.

Sone T (2011). Current methods in the diagnosis of osteoporosis. Nihon Rinsho 69: 1228-1232.

Suykens JAK and Vandewalle J (1999). Least squares support vector machine classifiers. Neural Process. Lett. 9: 293-300.

Unnanuntana A, Gladnick BP, Donnelly E and Lane JM (2010). The assessment of fracture risk. J. Bone Joint Surg. Am. 92: 743-753.

Vestergaard P, Rejnmark L and Mosekilde L (2005). Osteoporosis is markedly underdiagnosed: a nationwide study from Denmark. Osteoporos. Int. 16: 134-141.

Wark JD (1996). Osteoporotic fractures: background and prevention strategies. Maturitas 23: 193-207.

Wilkins MR, Gasteiger E, Tonella L, Ou K, et al. (1998). Protein identification with N and C-terminal sequence tags in proteome projects. J. Mol. Biol. 278: 599-608.

Zheng Y, Xu Y, Ye B, Lei J, et al. (2003). Prostate carcinoma tissue proteomics for biomarker discovery. Cancer 98: 2576-2582.

Genetics and Molecular Research 14 (4): 15285-15294 (2015) 